\title{
Unequal Error Protection for MIMO Systems with a Hybrid Structure
}

\author{
Guang-Hua Yang, Dongxu Shen and Victor O. K. Li \\ Department of Electrical and Electronic Engineering \\ The University of Hong Kong, Pokfulam Road, Hong Kong, China \\ Email: \{ghyang, dxshen, vli\}@eee.hku.hk
}

\begin{abstract}
We propose a hybrid multiple-input-multiple-output (MIMO) architecture to implement unequal error protection (UEP) for video delivery in MIMO systems. On the transmitter side, some of the transmit antennas are used to implement transmit diversity for delivering video data with high priority, while others are employed for spatial multiplexing to transmit video data with low priority. On the receiver side, a hybrid signal detection mechanism is adopted to separate and decode the mixed data. The goal is to exploit the diversity gain to provide better protection to the high priority data, while transmitting the low priority data with spatial multiplexing to achieve high data rate. Analysis and simulation results demonstrate that the proposed UEP mechanism significantly enhances the quality of video reception with a high spectrum efficiency.
\end{abstract} UEP.

Index Terms-MIMO, transmit diversity, spatial multiplexing,

\section{INTRODUCTION}

Multiple-input-multiple-output (MIMO) technique exploits multiple antennas at both ends of a wireless link, yielding significant improvements in both spectral efficiency and link reliability [1], [2]. In recent years, MIMO technology is being incorporated into wireless communication systems such as 3G, WiMAX, and 802.11n. An important application for such systems is video delivery. On the other hand, unequal error protection (UEP) [3] is efficient in delivering error-sensitive video over the error-prone wireless channels. Since video data can be divided into layers of high priority (HP) and low priority (LP), UEP may be used to provide better protection to HP data so that received video quality is better preserved under transmission errors.

In MIMO systems, the multiple transmit antennas can be used to either achieve higher data rate with spatial multiplexing (SM), or produce diversity gain with spatial diversity (SD). In this work, we propose a UEP method over a hybrid MIMO system in which SM and SD are combined together. On the transmitter side, some transmit antennas are employed to realize SD, for transmitting HP data. Other antennas are employed to achieve SM for LP data. At the receiver, a hybrid detection and decoding structure is designed to separate and decode the mixed data. With the hybrid structure, a higher level of protection is provided by SD to the HP data, and high system throughput is achieved by SM. Through computer simulations, we show that the proposed UEP scheme achieves significantly better performance than other system configurations.

This paper is organized as follows. In Section II, we provide the background of MIMO systems and UEP. In Section III, we present the UEP implementation in the hybrid MIMO structure. Simulation results are presented in Section IV. Conclusion is given in Section V.

\section{BACKGROUND}

\section{A. MIMO Systems}

In MIMO schemes, the benefits of having multiple antennas can be exploited in two ways: maximizing the spectrum efficiency by utilizing spatial multiplexing, and improving the link quality by exploiting spatial diversity.

In SM, data on each antenna is transmitted in an independent way [4]. In a MIMO system with $N$ transmit antennas and $M$ receive antennas, at most $\min (M, N)$ independent data streams can be simultaneously delivered [5], which greatly boosts spectrum efficiency.

The basic idea of SD is that the successful reception ratio increases with the number of uncorrelated antenna elements being used [1]. The diversity gain is related to the diversity order which is determined by the number of uncorrelated spatial branches available between the transmitter and the receiver. Space time coding (STC) is an effective technique to achieve SD. As an important class of STC, space time block coding (STBC) [6], [7] is widely used due to its simplicity and effectiveness. In this work, Alamouti's STBC scheme [6] is employed for SD. As pointed out in [7], it is the only STBC scheme which can achieve a coding rate of one for complex constellations.

Generally, to achieve the best performance, MIMO systems require the channel for each transmit-receive antenna pair to be flat and uncorrelated. However, with a properly designed equalizer in the time domain or with orthogonal frequency division multiplexing (OFDM), the flat fading requirement can be satisfied even for frequency selective fading channels. For STBC, the channel is further required to be quasi-static, i.e., the channel does not change in a single block, but can vary from block to block. This assumption generally holds for a system with moderate movements.

\section{B. UEP and Layered Video Coding}

UEP provides different levels of protection to different parts of the video data which may have unequal degrees of importance. Basically, UEP changes the distribution of errors without incurring extra resource consumption. Less bit errors are suffered by more important data. To achieve UEP, some 682 
layered video coding scheme need to be employed to code the video source into two or more layers with different priorities. With two layers, for example, the HP layer carries more vital data and can be decoded by itself to reconstruct the video with acceptable quality; the LP layer carries less important data which is used to improve the video quality. Errors in the HP layer have detrimental effects on the reconstructed video quality and should thus be avoided as much as possible. On the other hand, errors in the LP layer are more tolerable. Therefore, UEP aims to provide the best possible protection to the HP layer to achieve good video quality. Currently, layered coding is supported by major video compression standards, such as MPEG-2, H.263++, MPEG-4 and H.264. Data partitioning is the simplest form of layered coding. Compared with single-layer schemes, data partitioning combined with UEP provides considerably more error resilience with little extra complexity and overhead. In this paper, MPEG-2 [8] with data partitioning is adopted.

\section{UEP ON HYBRID MIMO SYSTEMS}

\section{A. Hybrid MIMO Structure}

In this paper, we exemplify our hybrid MIMO system with 4 by 4 antennas as presented in Fig. 1. It consists of a hybrid transmitter and a hybrid receiver.

1) Hybrid transmitter: The structure of the hybrid transmitter is quite simple. The 4 transmit antennas are designated into two groups: one group (2 antennas) is used for SD by using Alamouti's STBC scheme [6], and another group to transmit independent data in SM.

In STBC, the coding transform is performed on two consecutive time slots. Let $S_{11}$ and $S_{12}$ be the two input symbols of the STBC encoder. Then $S_{11}, S_{12}$ and $-S_{12}^{*}$ and $S_{11}^{*}$ are the encoded symbols, which are sent out from two SD antennas at slots 1 and 2, respectively. Therefore, only two data symbols are transmitted on the SD antennas during the two time slots. On the other hand, data transmitted through the SM antennas are independent, and correspondingly 4 symbols can be sent in two time slots, i.e., $S_{21}$ and $S_{22}$ on time slot 1 and $S_{23}$ and $S_{24}$ on slot 2. All the symbols transmitted in the two slots can be represented as

$$
\mathbf{S}=\left[\begin{array}{cc}
S_{11} & -S_{12}^{*} \\
S_{12} & S_{11}^{*} \\
S_{21} & S_{23} \\
S_{22} & S_{24}
\end{array}\right]
$$

where each row corresponds to a transmit antenna and each column corresponds to a time slot.

2) Hybrid receiver: The processing at the receiver is also performed on two consecutive time slots. Assuming the channels are uncorrelated and flat, and denoting $\mathbf{H}$ as the MIMO channel matrix with the dimension of $4 \times 4$, the received signal $\mathbf{R}$ on all receive antennas can be represented as

$$
\mathbf{R}=\mathbf{H S}+\mathbf{N}
$$

where $\mathbf{N}$ is additive white Gaussian noise. The detection and decoding process is performed on $\mathbf{R}$.
At the first step, a zero-forcing detection is employed to separate the mixed data streams. We assume $\mathbf{H}$ is known at the receiver, and let $\mathbf{G}$ denote the pseudo-inverse of $\mathbf{H}$ as

$$
\mathbf{G}=\left(\mathbf{H}^{*} \mathbf{H}\right)^{-1} \mathbf{H}^{*}
$$

where $(\cdot)^{*}$ represents the Hermitian transpose of a matrix. Then the zero-forcing detection is

$$
\hat{\mathbf{S}}=\mathbf{G R}=\mathbf{S}+\mathbf{G N} .
$$

Since the symbols transmitted with SM are independent in the spatial and time domains, the last two rows of $\hat{\mathbf{S}}$ are the estimated SM data $\hat{S_{2 k}}, k=1 \cdots 4$.

STBC data is decoded at the second step. Due to the interference among the multiple streams, the orthogonality condition for STBC decoding does not hold. Therefore, the interference of SM data should be removed before STBC decoding. This can be done by

$$
\mathbf{R}_{S T B C}=\mathbf{R}-\mathbf{H S}_{S M},
$$

where $\mathbf{S}_{S M}=\left[\begin{array}{cc}0 & 0 \\ 0 & 0 \\ \hat{S_{21}} & \hat{S_{23}} \\ \hat{S_{22}} & \hat{S_{24}}\end{array}\right]$ and $\hat{S_{2 k}}, k=1 \cdots 4$ are the estimated SM data (last two rows of $\hat{\mathbf{S}}$ ). Then $\mathbf{R}_{S T B C}$ is fed into a standard STBC decoder. Note that the STBC system has 2 transmit antennas and 4 receive antennas, i.e., all the received signals from the four antennas will be used for decoding.

3) Discussions and implementation issues: For simplicity, zero-forcing is employed at the receiver for signal detection. If other detection scheme, such as minimum mean squared error (MMSE), is employed, better detection performance can be expected with additional complexity.

In the above example, we consider a MIMO system with 4 by 4 antennas. For a MIMO system with more antennas, we can have more freedom in selecting the combinations of diversity and multiplexing schemes. For example, more antennas can be used for SM rather than SD, because the diversity gain has diminishing returns with increasing diversity order.

In order to focus on the effects of SD and SM, we do not employ any channel coding or interleaving scheme in our system. Of course, for a practical system, channel coding and interleaving are effective to achieve a better performance.

\section{B. UEP on Hybrid MIMO}

In the above hybrid MIMO systems, two antenna transmission modes, SM and SD, are utilized in a single system. Due to diversity gain, it can be expected that data transmitted with SD has better BER performance than that with SM. On the other hand, data transmitted with SM has a higher spectrum efficiency due to multiplexing. Such characteristics of hybrid MIMO can be utilized to realize UEP for video transmission.

Fig. 2 shows the schematic of the proposed UEP system (the block "PSNR calculator" is used for performance evaluation). At the transmitter, the source video sequence is coded and partitioned into an HP sub-stream and an LP sub-stream. With 

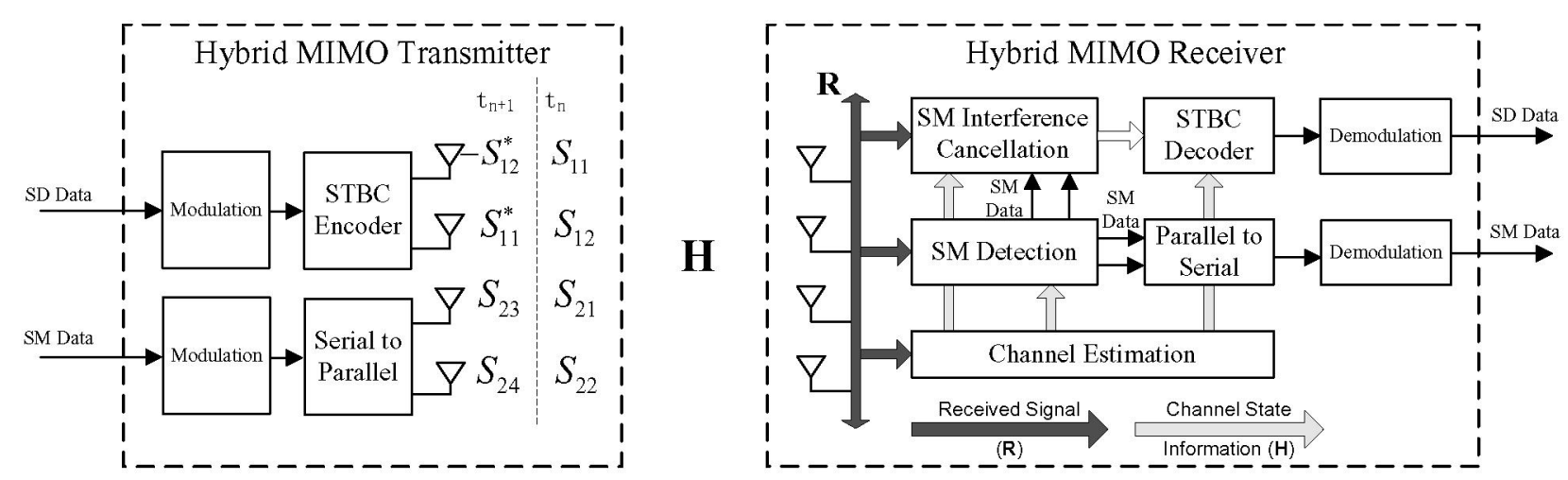

Fig. 1. Structure of a hybrid spatial diversity and spatial multiplexing system.

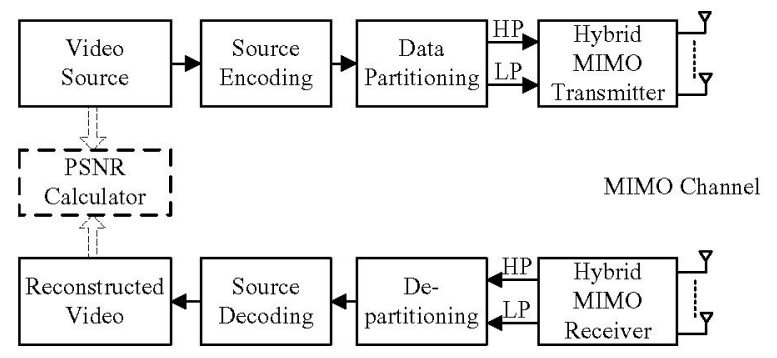

Fig. 2. UEP based on hybrid MIMO structure.

the hybrid MIMO, the HP data is transmitted by STBC, while the LP data is transmitted with SM. At the receiver, the data belonging to the two layers are separated and decoded. Then the data of two layers are combined and fed into the video decoder to reproduce the video sequence.

Such an UEP scheme benefits not only from the multiplexing gain, but also from the diversity gain. Obviously, HP video data experience less errors and LP video data achieve a high throughput. Thus better video quality can be achieved with high spectrum efficiency.

\section{Simulation Results}

In this section, the performance of the hybrid MIMO and the hybrid MIMO-based UEP scheme are evaluated by computer simulations. Besides the proposed hybrid MIMO structure, two other structures, namely, Twin-STBC (TS) and Full-Multiplexing (FM) shown in Fig. 3 (for simplicity, the structure of the receiver is omitted), are also evaluated for comparison. For TS, two modulation schemes, QPSK and 16QAM, are tested. The system parameters are listed in Table I. The scheme index will be used in the following parts for convenience. For all the simulations, a quasi-static Rayleigh fading channel is assumed.

\section{A. BER Performance Comparison}

The BER performance of all systems is shown in Fig. 4. For System A, two curves are shown for different data streams with SD and SM, respectively. We find that for System A, there is a significant BER difference between the data transmitted with SD and SM. SD data enjoys about $10 d B$ SNR gain
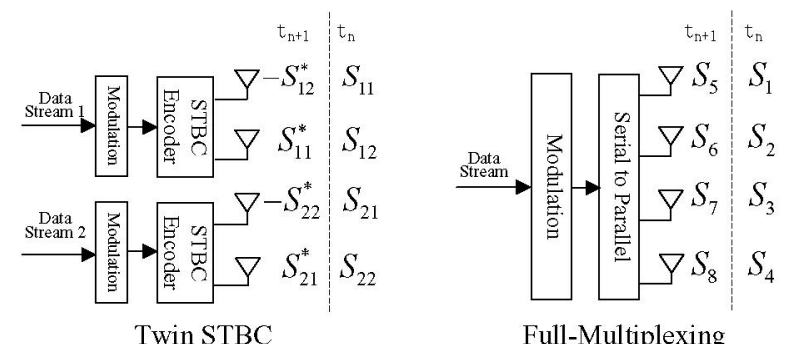

Full-Multiplexing

Fig. 3. Transmitter structure for $4 \times 4$ MIMO systems.

TABLE I

Simulated Systems.

\begin{tabular}{c|c|c|c}
\hline index & Structure & $\begin{array}{c}\text { Modulation } \\
\text { Scheme }\end{array}$ & $\begin{array}{c}\text { Relative } \\
\text { Data Rate }\end{array}$ \\
\hline \hline A & Hybrid STBC \& SM & QPSK & 3 \\
\hline B & Twin STBC & QPSK & 2 \\
\hline C & Twin STBC & 16 QAM & 4 \\
\hline D & Full-multiplexing & QPSK & 4 \\
\hline
\end{tabular}

over the SM data. System B has the same performance as achieved by the SD data of System A. Meanwhile, System D has the same performance as the SM data in System A. Thus the BER performance gain from SD is achieved without degrading the performance of SM data. This can be explained as follows. A MIMO channel matrix $\mathbf{H}$ with dimension $M \times N$ can be decomposed into $m, m \leq \min (M, N)$, equivalent parallel single-input-single-output (SISO) channels, where $m$ is the rank of $\mathbf{H}$ [9]. This gives us the freedom to tradeoff SM with diversity schemes. System C achieves slightly better performance than System D, and SM data in System A. It indicates that the diversity gain outweighs the extra SNR requirement for changing the modulation scheme from QPSK to 16 QAM.

\section{B. Performance of UEP on hybrid MIMO}

In this part, we evaluate the performance of the proposed UEP scheme based on System A. For comparison, three systems with no UEP based on Systems B, C and D are also evaluated. MPEG-2 [8] is employed as the video coding scheme. The standard video sequence "Mobile" is used as the 


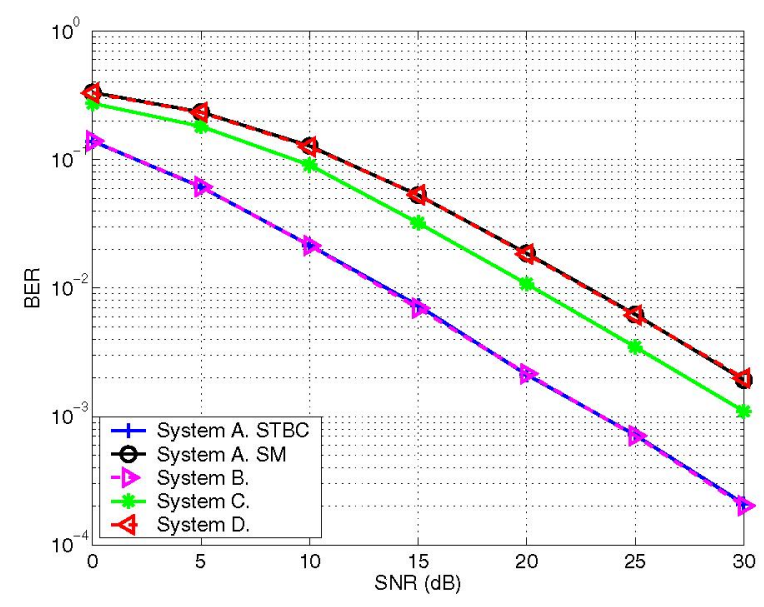

Fig. 4. BER performance of hybrid MIMO and other configurations.

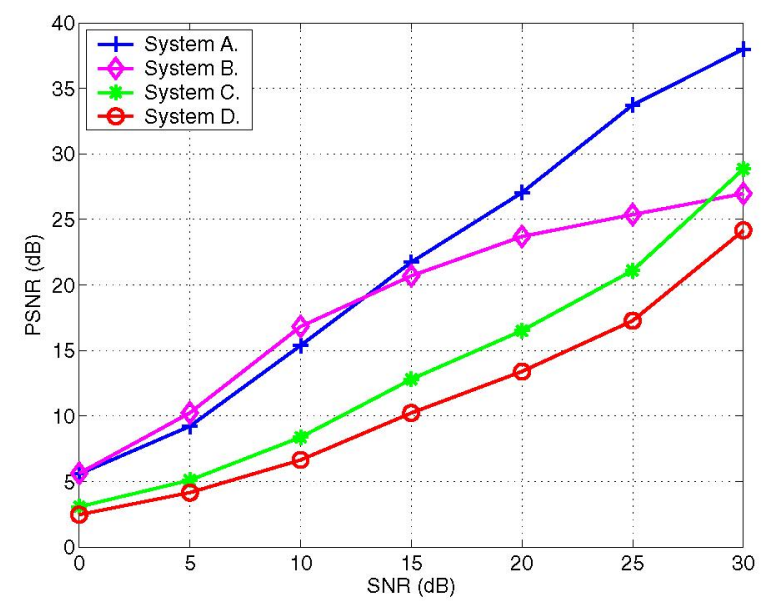

Fig. 5. UEP performance comparison (PSNR vs. SNR).

video source. This sequence is in the common intermediate format (CIF) with a resolution of $352 \times 288$, and a sampling ratio of $4: 2: 0$. Data partitioning is employed to partition the compressed video into data streams with different priorities. For a fair comparison, the source coding rate is adjusted according to the data rate of each system. That means all the systems transmit the video sequence with the same amount of time. Peak signal-to-noise ratio (PSNR) is calculated to evaluate the received video quality by comparing the output video with the source sequence. The simulation results are shown in Fig. 5.

From Fig. 5, we find that System A (the system index is used to denote the corresponding video transmission system) exhibits significantly better performance than Systems $C$ and $\mathrm{D}$ with $S N R<30 \mathrm{~dB}$. The PSNR gain is around $15 d B$ at $S N R=20 \mathrm{~dB}$. When the three systems have similar PSNR quality at $25 d B$, System A has about $10 d B$ SNR gain. System B has slightly better performance (less than $1 d B$ in SNR) than System A under low SNR $(S N R<13 d B)$. This is because System B provides equal protection to all video data, and thus LP data experience less errors than System A. However, System B outperforms System A only in the sub-20 $d B$ low PSNR range, which exhibits quite poor visual quality. For an acceptable quality of $P S N R>30 d B$, System A performs much better than any other systems, especially System B, whose PSNR curve saturates very undesirably with SNR increase. A consistently better performance can be observed for System C than System D. From the above, we can conclude that hybrid MIMO-based UEP achieves good tradeoff between quality and efficiency.

\section{COnClusion}

In this paper, we propose a hybrid UEP structure to combine spatial diversity and spatial multiplexing in a single MIMO system. The proposed hybrid MIMO structure achieves high spectrum efficiency with the ability to provide different levels of protection to video data with different priorities. The new UEP scheme achieves significant performance gain with respect to other MIMO configurations.

\section{ACKNOWLEDGMENTS}

This research is supported in part by the Research Grants Council of the Hong Kong Special Administrative Region, China (Project no. HKU 7152/05E).

\section{REFERENCES}

[1] D. Gesbert, M. Shafi, D. S. Shiu, P. J. Smith and A. Nauib, "From theory to practice: an overview of MIMO space-time coded wireless systems," IEEE J. Select. Areas Commun., Vol. 21, No. 3, April 2003.

[2] G. J. Foschini and M. J. Gans, "On limits of wireless communications in a fading environment when using multiple antennas," Wireless Pers. Commun., Vol. 6, No. 3, pp. 311-335, Mar. 1998.

[3] L. Hanzo, J. Streit, "Adaptive low-rate wireless video phone schemes," IEEE Trans. Circuits Syst. Video Technol., Vol. 5, No. 4, pp. 305-319, Aug. 1995.

[4] G. D. Golden, G. J. Foschini, R. A. Valenzuela, and P. W. Wolniansky, "Detection algorithm and initial laboratory results using the V-BLAST space-time communication architecture," Electron. Lett., Vol. 35, No. 1, pp. 14-15, 1999.

[5] G. J. Foschini, "Layered space-time architecture for wireless communication in a fading environment when using multi-element antennas," Bell Labs Tech. J., Vol. 1, No. 2, pp. 41-59, 1996.

[6] S. M. Alamouti, "A simple transmit diversity technique for wireless communications," IEEE J. Select. Areas Commun., Vol. 16, No. 8, pp. 1451-1458, Oct. 1998.

[7] V. Tarokh, H. Jafarkhani, A. R. Calderbank, "Space-time block codes from orthogonal designs," IEEE Trans. Inform. Theory, Vol. 45, No. 5, pp. 1456-1467, July 1999 .

[8] ISO/IEC 13818-2, Recommendation ITU-T H.262, 1995.

[9] E. Telatar, "Capacity of multi-antenna Gaussian channels," European Trans. Telecommun., Vol. 10, pp. 585-595, 1999. 